\title{
Diophantine Approximation with Mixed Powers of Primes
}

\author{
Huafeng Liu and Jing Huang*
}

\begin{abstract}
Let $k$ be an integer with $k \geq 3$. Let $\lambda_{1}, \lambda_{2}, \lambda_{3}$ be non-zero real numbers, not all negative. Assume that $\lambda_{1} / \lambda_{2}$ is irrational and algebraic. Let $\mathcal{V}$ be a well-spaced sequence, and $\delta>0$. In this paper, we prove that, for any $\varepsilon>0$, the number of $v \in \mathcal{V}$ with $v \leq X$ such that the inequality

$$
\left|\lambda_{1} p_{1}^{2}+\lambda_{2} p_{2}^{2}+\lambda_{3} p_{3}^{k}-v\right|<v^{-\delta}
$$

has no solution in primes $p_{1}, p_{2}, p_{3}$ does not exceed $O\left(X^{1-2 /\left(7 m_{2}(k)\right)+2 \delta+\varepsilon}\right)$, where $m_{2}(k)$ relies on $k$. This refines a recent result. Furthermore, we briefly describe how a similar method can refine a previous result on a Diophantine problem with two squares of primes, one cube of primes and one $k$-th power of primes.
\end{abstract}

\section{Introduction}

Diophantine approximation is an important topic in number theory. We first introduce the definition of a well-spaced sequence. We call an increasing sequence $v_{1}<v_{2}<\cdots$ of positive real numbers a well-spaced sequence if there exist positive constants $C>c>0$ such that

$$
0<c<v_{i+1}-v_{i}<C, \quad i=1,2, \ldots
$$

Let $k$ be an integer. In this paper, we first consider a Diophantine problem with two squares of primes and one $k$-th power of primes. Let

$$
m_{2}(k)= \begin{cases}4 & \text { if } k=3, \\ 2^{k / 2} & \text { if } k=4,6,8, \\ \frac{1}{2}\left(2^{(k-1) / 2}+2^{(k+1) / 2}\right) & \text { if } k=5,7,9, \\ \min \left(2^{[(k+1) / 2]},\left[\frac{k+1}{2}\right]\left(\left[\frac{k+1}{2}\right]+1\right)\right) & \text { if } k \geq 10,\end{cases}
$$

where $[x]$ denotes the greatest integer not exceeding $x$. Let $\lambda_{1}, \lambda_{2}, \lambda_{3}$ be non-zero real numbers, not all negative. Let $\mathcal{V}$ be a well-spaced sequence, and $\delta>0$. Let $E_{k}(\mathcal{V}, X, \delta)$ denote the number of $v \in \mathcal{V}$ with $v \leq X$ such that the inequality

$$
\left|\lambda_{1} p_{1}^{2}+\lambda_{2} p_{2}^{2}+\lambda_{3} p_{3}^{k}-v\right|<v^{-\delta}
$$

Received November 22, 2018; Accepted February 13, 2019.

Communicated by $\mathrm{Yu}-\mathrm{Ru} \mathrm{Liu}$.

2010 Mathematics Subject Classification. 11P32, 11P55, 11N36.

Key words and phrases. Waring-Goldbach problem, Diophantine inequality, Sieve methods.

*Corresponding author. 
has no solution in primes $p_{1}, p_{2}, p_{3}$. The inequality 1.2 with $k=2$ is considered in Harman [6]. Throughout this paper, constants, both explicit and implicit, in Vinogradov symbols may depend on $\lambda_{1}, \lambda_{2}, \lambda_{3}, \lambda_{4}$. We study the inequality (1.2) with $k \geq 3$ and prove the following theorems.

Theorem 1.1. Let $k$ be an integer with $k \geq 3$. Let $\lambda_{1}, \lambda_{2}, \lambda_{3}$ be non-zero real numbers, not all negative. Assume that $\lambda_{1} / \lambda_{2}$ is irrational and algebraic. Let $\mathcal{V}$ be a well-spaced sequence, and $\delta>0$. Then we have, for any $\varepsilon>0$,

$$
E_{k}(\mathcal{V}, X, \delta) \ll X^{1-2 /\left(7 m_{2}(k)\right)+2 \delta+\varepsilon},
$$

where $m_{2}(k)$ is defined by 1.1 .

Theorem 1.2. Let $k$ be an integer with $k \geq 3$. Let $\lambda_{1}, \lambda_{2}, \lambda_{3}$ be non-zero real numbers, not all negative. Assume that $\lambda_{1} / \lambda_{2}$ is irrational. Let $\mathcal{V}$ be a well-spaced sequence, and $\delta>0$. Then there is a sequence $X_{j} \rightarrow \infty$ such that, for any $\varepsilon>0$,

$$
E_{k}\left(\mathcal{V}, X_{j}, \delta\right) \ll X_{j}^{1-2 /\left(7 m_{2}(k)\right)+2 \delta+\varepsilon},
$$

where $m_{2}(k)$ is defined by (1.1). Moreover, if the convergent denominators $q_{j}$ for $\lambda_{1} / \lambda_{2}$ satisfy

$$
q_{j+1}^{1-w} \ll q_{j} \quad \text { for some } w \in[0,1)
$$

then we have, for all $X \geq 1$ and any $\varepsilon>0$,

$$
E_{k}(\mathcal{V}, X, \delta) \ll X^{1-(2-4 \chi) / m_{2}(k)+2 \delta+\varepsilon}
$$

with

$$
\chi=\max \left(\frac{5-3 w+2 / m_{2}(k)}{12-8 w+4 / m_{2}(k)}, \frac{3}{7}\right) .
$$

Results of this type were first obtained by Ge and Wang [5]. Let

$$
\sigma(k)=\min \left(2^{[(k+1) / 2]-1}, \frac{1}{2}\left[\frac{k+1}{2}\right]\left(\left[\frac{k+1}{2}\right]+1\right)\right) .
$$

They proved that

$$
\begin{aligned}
E_{k}(\mathcal{V}, X, \delta) & \ll X^{1-1 /(8 \sigma(k))+2 \delta+\varepsilon}, \\
E_{k}\left(\mathcal{V}, X_{j}, \delta\right) & \ll X_{j}^{1-1 /(8 \sigma(k))+2 \delta+\varepsilon}, \\
E_{k}(\mathcal{V}, X, \delta) & \ll X^{1-(1-\chi) / \sigma(k)+2 \delta+\varepsilon}
\end{aligned}
$$


with

$$
\chi=\max \left(\frac{5+1 / \sigma(k)-3 w}{6+1 / \sigma(k)-4 w}, \frac{7}{8}\right),
$$

in replace of $(1.3),(1.4), 1.6$ and $(1.7)$, respectively. It is easy to verify that the results in Theorems 1.1 and 1.2 improve Ge and Wang's results.

Theorem 1.1 follows immediately from Theorem 1.2 , since, in the case of $\lambda_{1} / \lambda_{2}$ algebraic, we can take $w=\varepsilon$.

To prove Theorem 1.2, we apply the Davenport-Heilbronn version of the HardyLittlewood method. On the one hand, the improvement not only derives from more carefully estimating the integral by using an optimal choice of different Hölder's inequalities from [5], but also the recent new breakthrough of Vinogradov's mean value theorem [1]. On the other hand, motivated by the work of Wang and Yao [13], we use the sieve functions $\rho^{ \pm}(m)$ constructed in Harman [6], which also results in the improvement.

Many authors also considered the inequality

$$
\left|\lambda_{1} p_{1}^{2}+\lambda_{2} p_{2}^{2}+\lambda_{3} p_{3}^{3}+\lambda_{4} p_{4}^{k}-v\right|<v^{-\delta} .
$$

Let $\lambda_{1}, \lambda_{2}, \lambda_{3}, \lambda_{4}$ be positive real numbers. Assume that $\lambda_{1} / \lambda_{2}$ is irrational and algebraic. Let $\mathcal{V}$ be a well-spaced sequence, and $\delta>0$. Let $E_{k}^{*}(\mathcal{V}, X, \delta)$ denote the number of $v \in \mathcal{V}$ with $v \leq X$ such that the inequality (1.8) has no solution in primes $p_{1}, p_{2}, p_{3}, p_{4}$. In 2010, Li and Wang [8] proved that, for any $\varepsilon>0$,

$$
E_{3}^{*}(\mathcal{V}, X, \delta) \ll X^{20 / 21+2 \delta+\varepsilon} .
$$

Subsequently, the exponent 20/21 was improved to 67/72 by $\mathrm{Mu}$ and $\mathrm{Lü} 9$. Recently $\mathrm{Mu}$ and Lü [10] refined the exponent to 29/33. In the same paper [10], Mu and Lü also considered the case $k \geq 4$ and proved that

$$
E_{k}^{*}(\mathcal{V}, X, \delta) \ll \begin{cases}X^{1-1 / 11+2 \delta+\varepsilon} & \text { if } k=4 \\ X^{1-2 /(11 k)-16 /\left(11 k^{2}(k+1)\right)+2 \delta+\varepsilon} & \text { if } k \geq 5\end{cases}
$$

It is not hard to show that the argument proving Theorem 1.2 leads also to improvements on the results established by $\mathrm{Mu}$ and Lü 10 . Let

$$
m_{3}(k)= \begin{cases}2^{[(k-1) / 6]+1} & \text { if } 5 \leq k \leq 48 \\ 2\left[\frac{k^{2}+k+6}{12}\right] & \text { if } k \geq 49\end{cases}
$$

We have the following theorem.

Theorem 1.3. Let $k$ be an integer with $k \geq 3$. Let $\lambda_{1}, \lambda_{2}, \lambda_{3}, \lambda_{4}$ be positive real numbers. Assume that $\lambda_{1} / \lambda_{2}$ is irrational and algebraic. Let $\mathcal{V}$ be a well-spaced sequence, and $\delta>0$. Then we have, for any $\varepsilon>0$,

$$
E_{k}^{*}(\mathcal{V}, X, \delta) \ll X^{1-\sigma^{*}(k)+2 \delta+\varepsilon},
$$


where

$$
\sigma^{*}(k)= \begin{cases}1 / 7 & \text { if } k=3,4, \\ 1 / 14+1 /\left(7 m_{3}(k)\right) & \text { if } 5 \leq k \leq 48, \\ 1 / 14+2 /\left(7 m_{2}(k)\right) & \text { if } k \geq 49,\end{cases}
$$

and $m_{2}(k), m_{3}(k)$ are defined by 1.1 and $(1.9)$, respectively.

Since the proof of Theorem 1.3 is similar to that of Theorem 1.2 , we prove Theorem 1.2 in the following Sections 26 and sketch the proof of Theorem 1.3 in Section 7.

Notation. Throughout this paper, the letter $p$, with or without a subscript, always denotes a prime. We use $\varepsilon$ to denote a sufficiently small positive number, and the value of $\varepsilon$ may change from statement to statement. We abbreviate $\log x$ to $L$.

\section{Outline of the method}

To use the Davenport-Heilbronn method, we first introduce some notations. Suppose that $k \geq 3$ is an integer and $\eta$ is a fixed sufficiently small positive number. Let $0<\tau<1$, indeed we shall chose $\tau=X^{-\delta}$ in Section [6. Let

$$
K_{\tau}(\alpha)=\left(\frac{\sin (\pi \tau \alpha)}{\pi \alpha}\right)^{2}
$$

for $\tau>0$ and $\alpha \neq 0$. By continuity, we define $K_{\tau}(0)=\tau^{2}$. Then we have

$$
K_{\tau}(\alpha) \ll \min \left(\tau^{2},|\alpha|^{-2}\right),
$$

and

$$
\widehat{K}_{\tau}(t):=\int_{\mathbb{R}} e(t \alpha) K_{\tau}(\alpha) d \alpha=\max (0, \tau-|t|)
$$

Assume that $a / q$ is a convergent to $\lambda_{1} / \lambda_{2}$, with the denominator $q$ sufficiently large. Fix $X=q^{7 / 3}$. We write

$$
I_{1}=\left[\left(\frac{\eta X}{\lambda_{1}}\right)^{1 / 2},\left(\frac{2 \eta X}{\lambda_{1}}\right)^{1 / 2}\right], I_{2}=\left[\left(\frac{\eta X}{\lambda_{2}}\right)^{1 / 2},\left(\frac{2 \eta X}{\lambda_{2}}\right)^{1 / 2}\right], I_{3}=\left[\left(\frac{\eta X}{\lambda_{3}}\right)^{1 / k},\left(\frac{2 \eta X}{\lambda_{3}}\right)^{1 / k}\right] .
$$

Let $\rho_{0}(m)$ denote the characteristic function of the set of primes. To prove Theorem 1.2 , we suppose that we have arithmetic functions $\rho^{ \pm}(m)$ such that, for $m \in I_{i}, i=1,2$,

$$
\rho^{-}(m) \leq \rho_{0}(m) \leq \rho^{+}(m)
$$

Our choice of $\rho^{ \pm}(m)$ is borrowed from Harman $[6]$. Namely, $\rho^{-}$and $\rho^{+}$are the functions $b_{0}$ and $b_{1}$ constructed in Section 8 of [6], respectively. Here we just state some properties 
of the sieve functions $\rho^{ \pm}(m)$, one can refer to [6] (see also [7]) for their construction in detail. In many ways, the functions $\rho^{ \pm}(m)$ imitate the characteristic functions of primes. In particular, as in Section 8 of [6], for any subinterval $\mathcal{I} \in I_{i}, i=1,2$, one has

$$
\sum_{m \in \mathcal{I}} \rho^{ \pm}(m)=\kappa_{i}^{ \pm}|\mathcal{I}| L^{-1}+O\left(X^{1 / 2} L^{-2}\right)
$$

where $\kappa_{i}^{ \pm}>0$ are absolute constants satisfying

$$
\kappa_{i}^{-}>0.9, \quad \kappa_{i}^{+}<1.7, \quad i=1,2 .
$$

Then the vector sieve of Brüdern and Fouvry [2] gives

$$
\rho_{0}\left(m_{1}\right) \rho_{0}\left(m_{2}\right) \geq \rho^{-}\left(m_{1}\right) \rho^{+}\left(m_{2}\right)+\rho^{+}\left(m_{1}\right) \rho^{-}\left(m_{2}\right)-\rho^{+}\left(m_{1}\right) \rho^{+}\left(m_{2}\right) \text {. }
$$

We define

$$
S_{1}(\alpha, \rho)=\sum_{m \in I_{1}} \rho(m) e\left(m^{2} \alpha\right), \quad S_{2}(\alpha, \rho)=\sum_{m \in I_{2}} \rho(m) e\left(m^{2} \alpha\right), \quad S_{k}(\alpha)=\sum_{p \in I_{3}}(\log p) e\left(p^{k} \alpha\right) .
$$

For any measurable subset $\mathfrak{X}$ of $\mathbb{R}$, write

$$
I\left(\tau, v, \mathfrak{X}, \rho_{1}, \rho_{2}\right)=\int_{\mathfrak{X}} S_{1}\left(\lambda_{1} \alpha, \rho_{1}\right) S_{2}\left(\lambda_{2} \alpha, \rho_{2}\right) S_{k}\left(\lambda_{3} \alpha\right) e(-\alpha v) K_{\tau}(\alpha) d \alpha .
$$

Then from 2.2 , we have

$$
\begin{aligned}
I\left(\tau, v, \mathbb{R}, \rho_{0}, \rho_{0}\right)= & \sum_{\substack{m_{i} \in I_{i}, i=1,2 \\
p_{3} \in I_{3}}} \rho_{0}\left(m_{1}\right) \rho_{0}\left(m_{2}\right) \log p_{3} \\
& \times \int_{-\infty}^{\infty} e\left(\left(\lambda_{1} m_{1}^{2}+\lambda_{2} m_{2}^{2}+\lambda_{3} p_{3}^{k}-v\right) \alpha\right) K_{\tau}(\alpha) d \alpha \\
= & \sum_{\substack{m_{i} \in I_{i}, i=1,2 \\
p_{3} \in I_{3}}} \rho_{0}\left(m_{1}\right) \rho_{0}\left(m_{2}\right) \log p_{3} \\
& \times \max \left(0, \tau-\left|\lambda_{1} m_{1}^{2}+\lambda_{2} m_{2}^{2}+\lambda_{3} p_{3}^{k}-v\right|\right) .
\end{aligned}
$$

Thus we have

$$
I\left(\tau, v, \mathbb{R}, \rho_{0}, \rho_{0}\right) \ll \tau \log X \mathfrak{N}(X),
$$

where $\mathfrak{N}(\mathrm{X})$ denotes the number of solutions of the inequality

$$
\left|\lambda_{1} p_{1}^{2}+\lambda_{2} p_{2}^{2}+\lambda_{3} p_{3}^{k}-v\right|<\tau
$$

with $p_{j} \in I_{j}$ for $1 \leq j \leq 3$. Recalling $(2.5)$, we have

$$
I\left(\tau, v, \mathbb{R}, \rho_{0}, \rho_{0}\right) \geq I\left(\tau, v, \mathbb{R}, \rho^{-}, \rho^{+}\right)+I\left(\tau, v, \mathbb{R}, \rho^{+}, \rho^{-}\right)-I\left(\tau, v, \mathbb{R}, \rho^{+}, \rho^{+}\right) .
$$


Then it is sufficient to give a positive lower bound for the right side of (2.6). Due to a dyadic dissection argument, we focus on those $v$ such that $X / 2 \leq v \leq X$. To do this, we divide the real line into the major arc $\mathfrak{M}$, the minor arc $\mathfrak{m}$ and the trivial arc $\mathfrak{t}$. We define

$$
\mathfrak{M}=\{\alpha:|\alpha| \leq P / X\}, \quad \mathfrak{m}=\{\alpha: P / X<|\alpha| \leq R\}, \quad \mathfrak{t}=\{\alpha:|\alpha|>R\},
$$

where $P=X^{1 / 4}$ and $R=\tau^{-2} X^{1 / 2+2 \varepsilon}$. Thus we can write

$$
I\left(\tau, v, \mathbb{R}, \rho_{1}, \rho_{2}\right)=I\left(\tau, v, \mathfrak{M}, \rho_{1}, \rho_{2}\right)+I\left(\tau, v, \mathfrak{m}, \rho_{1}, \rho_{2}\right)+I\left(\tau, v, \mathfrak{t}, \rho_{1}, \rho_{2}\right) .
$$

We write

$$
H(\alpha)=S_{1}\left(\lambda_{1} \alpha, \rho^{-}\right) S_{2}\left(\lambda_{2} \alpha, \rho^{+}\right)+S_{1}\left(\lambda_{1} \alpha, \rho^{+}\right) S_{2}\left(\lambda_{2} \alpha, \rho^{-}\right)-S_{1}\left(\lambda_{1} \alpha, \rho^{+}\right) S_{2}\left(\lambda_{2} \alpha, \rho^{+}\right) .
$$

We estimate the integrals on the major arc, the minor arc and the trivial arc in the following Sections 3 , 4 and 5, respectively. In Section 6, we complete the proof of Theorem 1.2 .

\section{The major arc}

In this section, we estimate the contribution from the right side of (2.6) on the major arc. Since the methods of estimating $I\left(\tau, v, \mathfrak{M}, \rho^{-}, \rho^{+}\right), I\left(\tau, v, \mathfrak{M}, \rho^{+}, \rho^{-}\right)$and $I\left(\tau, v, \mathfrak{M}, \rho^{+}, \rho^{+}\right)$ are similar, we can focus on $I\left(\tau, v, \mathfrak{M}, \rho^{-}, \rho^{+}\right)$in the following. As in [5], we first consider the standard major arc $\widetilde{\mathfrak{M}}=\left\{\alpha:|\alpha| \leq \phi=X^{-1+5 /(6 k)-\varepsilon}\right\}$. Let

$$
T_{1}(\alpha)=\int_{I_{1}} e\left(t^{2} \alpha\right) d t, \quad T_{2}(\alpha)=\int_{I_{2}} e\left(t^{2} \alpha\right) d t, \quad T_{k}(\alpha)=\int_{I_{3}} e\left(t^{k} \alpha\right) d t .
$$

Then, using a trivial bound for $S_{j}$, and the first derivative estimate for trigonometric integrals (see Titchmarsh [11, Lemma 4.2]), one has

$$
\begin{array}{rlrl}
S_{1}\left(\alpha, \rho^{-}\right) & \ll X^{1 / 2}, & & T_{1}(\alpha) \ll X^{1 / 2-1} \min \left(X,|\alpha|^{-1}\right), \\
S_{2}\left(\alpha, \rho^{+}\right) \ll X^{1 / 2}, & & T_{2}(\alpha) \ll X^{1 / 2-1} \min \left(X,|\alpha|^{-1}\right), \\
S_{k}(\alpha) \ll X^{1 / k}, & & T_{k}(\alpha) \ll X^{1 / k-1} \min \left(X,|\alpha|^{-1}\right) .
\end{array}
$$

Then we can rewrite $I\left(\tau, v, \widetilde{\mathfrak{M}}, \rho^{-}, \rho^{+}\right)$as follows:

$$
\begin{aligned}
& I\left(\tau, v, \widetilde{\mathfrak{M}}, \rho^{-}, \rho^{+}\right) \\
= & \kappa_{1}^{-} \kappa_{2}^{+} L^{-2} \int_{\widetilde{\mathfrak{M}}} T_{1}\left(\lambda_{1} \alpha\right) T_{2}\left(\lambda_{2} \alpha\right) T_{k}\left(\lambda_{3} \alpha\right) e(-\alpha v) K_{\tau}(\alpha) d \alpha \\
& +\kappa_{2}^{+} L^{-1} \int_{\widetilde{\mathfrak{M}}}\left(S_{1}\left(\lambda_{1} \alpha, \rho^{-}\right)-\kappa_{1}^{-} L^{-1} T_{1}\left(\lambda_{1} \alpha\right)\right) T_{2}\left(\lambda_{2} \alpha\right) T_{k}\left(\lambda_{3} \alpha\right) e(-\alpha v) K_{\tau}(\alpha) d \alpha \\
& +\int_{\widetilde{\mathfrak{M}}} S_{1}\left(\lambda_{1} \alpha, \rho^{-}\right)\left(S_{2}\left(\lambda_{2} \alpha, \rho^{+}\right)-\kappa_{2}^{+} L^{-1} T_{2}\left(\lambda_{2} \alpha\right)\right) T_{k}\left(\lambda_{3} \alpha\right) e(-\alpha v) K_{\tau}(\alpha) d \alpha \\
& +\int_{\widetilde{\mathfrak{M}}} S_{1}\left(\lambda_{1} \alpha, \rho^{-}\right) S_{2}\left(\lambda_{2} \alpha, \rho^{+}\right)\left(S_{k}\left(\lambda_{3} \alpha\right)-T_{k}\left(\lambda_{3} \alpha\right)\right) e(-\alpha v) K_{\tau}(\alpha) d \alpha \\
:= & J+J_{1}+J_{2}+J_{3} .
\end{aligned}
$$


In the following, we restrict our attention to estimate $J, J_{1}$ and $J_{2}$, since the computation for $J_{3}$ is similar to the corresponding one in Ge and Wang [5]. We first establish the lower bound for $J$. Note that

$$
\begin{aligned}
J= & \kappa_{1}^{-} \kappa_{2}^{+} L^{-2} \int_{\mathbb{R}} T_{1}\left(\lambda_{1} \alpha\right) T_{2}\left(\lambda_{2} \alpha\right) T_{k}\left(\lambda_{3} \alpha\right) e(-\alpha v) K_{\tau}(\alpha) d \alpha \\
& +O\left(L^{-1} \int_{|\alpha|>\phi} T_{1}\left(\lambda_{1} \alpha\right) T_{2}\left(\lambda_{2} \alpha\right) T_{k}\left(\lambda_{3} \alpha\right) e(-\alpha v) K_{\tau}(\alpha) d \alpha\right) .
\end{aligned}
$$

Putting this together with $(2.1)$ and $(3.2)$, we obtain that the error term in $(3.4)$ is

$$
\ll \tau^{2} X^{1 / k-2} \int_{|\alpha|>\phi} \frac{d \alpha}{|\alpha|^{3}} \ll \tau^{2} X^{1 / k-5 /(3 k)+2 \varepsilon}=o\left(\tau^{2} X^{1 / k} L^{-2}\right) .
$$

We write

$$
\begin{aligned}
f(v) & =\int_{\mathbb{R}} T_{1}\left(\lambda_{1} \alpha\right) T_{2}\left(\lambda_{2} \alpha\right) T_{k}\left(\lambda_{3} \alpha\right) e(-\alpha v) K_{\tau}(\alpha) d \alpha \\
& =\int_{I_{1} \times I_{2} \times I_{3}} \max \left(0, \tau-\left|\lambda_{1} t_{1}^{2}+\lambda_{2} t_{2}^{2}+\lambda_{3} t_{3}^{k}-v\right|\right) d t_{1} d t_{2} d t_{3} .
\end{aligned}
$$

Thus from (3.4), (3.5) and (3.6), we get

$$
J=f(v) \kappa_{1}^{-} \kappa_{2}^{+}(1+o(1)) L^{-2} .
$$

Now we turn to establish the upper bound for the integral $J_{1}$. Using (2.1), we have

$$
J_{1} \ll \tau^{2} L^{-1} \int_{\widetilde{\mathfrak{M}}}\left|S_{1}\left(\lambda_{1} \alpha, \rho^{-}\right)-\kappa_{1}^{-} L^{-1} T_{1}\left(\lambda_{1} \alpha\right)\right|\left|T_{2}\left(\lambda_{2} \alpha\right)\right|\left|T_{k}\left(\lambda_{3} \alpha\right)\right| d \alpha .
$$

By partial summation, we obtain

$$
S_{1}\left(\lambda_{1} \alpha, \rho^{-}\right)=\int_{I_{1}} e\left(\lambda_{1} t^{2} \alpha\right) d\left(\sum_{m \leq t, m \in I_{1}} \rho^{-}(m)\right) .
$$

From 2.3$)$, we have

$$
\left|S_{1}\left(\lambda_{1} \alpha, \rho^{-}\right)-\kappa_{1}^{-} L^{-1} T_{1}\left(\lambda_{1} \alpha\right)\right| \ll X^{1 / 2} L^{-2}(1+|\alpha| X) .
$$

Inserting (3.9) into (3.8), we obtain

$$
\begin{aligned}
J_{1} \ll & \tau^{2} X^{1 / 2} L^{-3} \int_{0}^{1 / X}\left|T_{2}\left(\lambda_{2} \alpha\right)\right|\left|T_{k}\left(\lambda_{3} \alpha\right)\right| d \alpha \\
& +\tau^{2} X^{3 / 2} L^{-3} \int_{1 / X}^{\phi}|\alpha|\left|T_{2}\left(\lambda_{2} \alpha\right)\right|\left|T_{k}\left(\lambda_{3} \alpha\right)\right| d \alpha .
\end{aligned}
$$

Then from (3.2), we obtain

$$
J_{1}=o\left(\tau^{2} X^{1 / k} L^{-2}\right)
$$


Arguing similarly, in spite of different sieve functions, we can also get

$$
J_{2}=o\left(\tau^{2} X^{1 / k} L^{-2}\right)
$$

Following the argument in [5, Section 3.3], we can obtain

$$
J_{3}=o\left(\tau^{2} X^{1 / k} L^{-2}\right)
$$

Therefore combining (3.3), 3.7), 3.10, 3.11) and (3.12), we have

$$
I\left(\tau, v, \widetilde{\mathfrak{M}}, \rho^{-}, \rho^{+}\right)=f(v) \kappa_{1}^{-} \kappa_{2}^{+}(1+o(1)) L^{-2} .
$$

Arguing similarly, we can get

$$
I\left(\tau, v, \widetilde{\mathfrak{M}}, \rho^{+}, \rho^{-}\right)=f(v) \kappa_{1}^{+} \kappa_{2}^{-}(1+o(1)) L^{-2}
$$

and

$$
I\left(\tau, v, \widetilde{\mathfrak{M}}, \rho^{+}, \rho^{+}\right)=f(v) \kappa_{1}^{+} \kappa_{2}^{+}(1+o(1)) L^{-2}
$$

From (3.5) in 5] or by a standard argument (see [3, Lemma 51]), we have

$$
f(v) \gg \tau^{2} X^{1 / k}
$$

Thus from (3.13), 3.14) and (3.15), we have

$$
\int_{\widetilde{\mathfrak{M}}} H(\alpha) S_{k}\left(\lambda_{3} \alpha\right) e(-\alpha v) K_{\tau}(\alpha) d \alpha \gg\left(\kappa_{1}^{-} \kappa_{2}^{+}+\kappa_{1}^{+} \kappa_{2}^{-}-\kappa_{1}^{+} \kappa_{2}^{+}+o(1)\right) \tau^{2} X^{1 / k} L^{-2} .
$$

Recalling 2.4 , since $1.7 \times 0.9+0.9 \times 1.7-1.7 \times 1.7>0$, we know that the coefficient in the right side of (3.1) is positive.

Note that $\widetilde{\mathfrak{M}} \supseteq \mathfrak{M}$ when $k=3$. The remaining region needed to be handled is $\mathfrak{M} \backslash \widetilde{\mathfrak{M}}$ for $k \geq 4$. Although there are sieve functions here, following a similar argument to [5. Lemma 3.3], we can get, for $k \geq 4$,

$$
\int_{\mathfrak{M} \backslash \tilde{\mathfrak{M}}}\left|H(\alpha) S_{k}\left(\lambda_{3} \alpha\right)\right| K_{\tau}(\alpha) d \alpha \ll \tau^{2} X^{1 / k-4^{1-k} /(2 k)+\varepsilon}=o\left(\tau^{2} X^{1 / k} L^{-2}\right)
$$

Therefore from the above analysis, we can conclude the following lemma.

Lemma 3.1. We have

$$
\int_{\mathfrak{M}} H(\alpha) S_{k}\left(\lambda_{3} \alpha\right) e(-\alpha v) K_{\tau}(\alpha) d \alpha \gg \tau^{2} X^{1 / k} L^{-2} .
$$




\section{The minor arc}

In this section, we give the estimate of the integral on the minor arc. Note that

$$
\begin{aligned}
& \int_{\mathfrak{m}}\left|H(\alpha) S_{k}\left(\lambda_{3} \alpha\right)\right|^{2} K_{\tau}(\alpha) d \alpha \\
\ll & \int_{\mathfrak{m}}\left|S_{1}\left(\lambda_{1} \alpha, \rho^{-}\right) S_{2}\left(\lambda_{2} \alpha, \rho^{+}\right) S_{k}\left(\lambda_{3} \alpha\right)\right|^{2} K_{\tau}(\alpha) d \alpha \\
& +\int_{\mathfrak{m}}\left|S_{1}\left(\lambda_{1} \alpha, \rho^{+}\right) S_{2}\left(\lambda_{2} \alpha, \rho^{-}\right) S_{k}\left(\lambda_{3} \alpha\right)\right|^{2} K_{\tau}(\alpha) d \alpha \\
& +\int_{\mathfrak{m}}\left|S_{1}\left(\lambda_{1} \alpha, \rho^{+}\right) S_{2}\left(\lambda_{2} \alpha, \rho^{+}\right) S_{k}\left(\lambda_{3} \alpha\right)\right|^{2} K_{\tau}(\alpha) d \alpha .
\end{aligned}
$$

From [6, Section 8], we know that the sieve functions can be expressed in terms of finitely many sums of the form

$$
\sum_{m=r s} f_{r} g_{s}
$$

where either $X^{1 / 7} \leq r \leq X^{3 / 14}$ or $f_{r} \equiv 1$ and $r \geq X^{1 / 7}$. In either case, $f_{r}, g_{s}$ are bounded by divisor functions at worst.

We write

$$
S_{i}(\alpha)=\sum_{n \in I_{i}} a_{n} e\left(n^{2} \alpha\right), \quad i=1,2,
$$

where $a_{n}$ takes the form 4.2. Without loss of generality we need only to consider the integral

$$
\int_{\mathfrak{m}}\left|S_{1}\left(\lambda_{1} \alpha\right) S_{2}\left(\lambda_{2} \alpha\right) S_{k}\left(\lambda_{3} \alpha\right)\right|^{2} K_{\tau}(\alpha) d \alpha
$$

First we need the following lemmas.

Lemma 4.1. Suppose that $X^{1 / 2} \geq Z \geq X^{3 / 7+\varepsilon / 2}$ and $\left|S_{j}\left(\lambda_{j} \alpha\right)\right|>Z$ for $j=1,2$. Then there are coprime integers $a_{j}, q_{j}$ satisfying

$$
1 \leq q_{j} \ll\left(\frac{X^{1 / 2+\varepsilon / 2}}{Z}\right)^{4}, \quad\left|q_{j} \lambda_{j} \alpha-a_{j}\right| \ll X^{-1}\left(\frac{X^{1 / 2+\varepsilon / 2}}{Z}\right)^{4} .
$$

Proof. This lemma is Lemma 1 in 13 .

Lemma 4.2. Let $m_{2}(k)$ and $m_{3}(k)$ be defined by (1.1) and (1.9), respectively. Suppose that $F \in\left\{S_{1}^{4}, S_{2}^{4}, S_{3}^{8}, S_{2}^{2} S_{k}^{m_{2}(k)}, S_{2}^{2} S_{3}^{2} S_{k}^{m_{3}(k)}\right\}$. Then we have

$$
\int_{-1}^{1}|F(\alpha)| d \alpha \ll X^{-1}(F(0))^{1+\varepsilon}, \quad \int_{-\infty}^{\infty}|F(\alpha)| K_{\tau}(\alpha) d \alpha \ll \tau X^{-1}(F(0))^{1+\varepsilon} .
$$


Proof. All of these results follow from [12 by using Hua's lemma and the recent breakthrough of Bourgain-Demeter-Guth [1] on Vinogradov's mean value theorem. We can find this result in [4, Lemma 3] for $3 \leq k \leq 9$ and in [5, Lemma 5.1] for $k \geq 10$, respectively.

Let $\widetilde{\mathfrak{m}}=\mathfrak{m}_{1} \cup \mathfrak{m}_{2}$, where

$$
\mathfrak{m}_{j}=\left\{\alpha \in \mathfrak{m}:\left|S_{j}\left(\lambda_{j} \alpha\right)\right| \leq X^{3 / 7+\varepsilon}\right\} \quad \text { for } j=1,2
$$

Lemma 4.3. For $k \geq 3$, we have

$$
\int_{\widetilde{\mathfrak{m}}}\left|S_{1}\left(\lambda_{1} \alpha\right) S_{2}\left(\lambda_{2} \alpha\right) S_{k}\left(\lambda_{3} \alpha\right)\right|^{2} K_{\tau}(\alpha) d \alpha \ll \tau X^{1+2 / k-2 /\left(7 m_{2}(k)\right)+\varepsilon},
$$

where $m_{2}(k)$ is defined by (1.1).

Proof. Using Hölder's inequality and Lemma 4.2, we can obtain

$$
\begin{aligned}
& \int_{\mathfrak{m}_{1}}\left|S_{1}\left(\lambda_{1} \alpha\right) S_{2}\left(\lambda_{2} \alpha\right) S_{k}\left(\lambda_{3} \alpha\right)\right|^{2} K_{\tau}(\alpha) d \alpha \\
\ll & \left(\sup _{\alpha \in \mathfrak{m}_{1}}\left|S_{1}\left(\lambda_{1} \alpha\right)\right|\right)^{4 / m_{2}(k)}\left(\int_{\mathbb{R}}\left|S_{1}\left(\lambda_{1} \alpha\right)\right|^{4} K_{\tau}(\alpha) d \alpha\right)^{1 / 2-1 / m_{2}(k)} \\
& \times\left(\int_{\mathbb{R}}\left|S_{2}\left(\lambda_{2} \alpha\right)\right|^{4} K_{\tau}(\alpha) d \alpha\right)^{1 / 2-1 / m_{2}(k)}\left(\int_{\mathbb{R}}\left|S_{2}\left(\lambda_{2} \alpha\right)\right|^{2}\left|S_{k}\left(\lambda_{3} \alpha\right)\right|^{m_{2}(k)} K_{\tau}(\alpha) d \alpha\right)^{2 / m_{2}(k)} \\
\ll & \tau X^{1+2 / k-2 /\left(7 m_{2}(k)\right)+\varepsilon} .
\end{aligned}
$$

By symmetry we can get the same bound for the integral on $\mathfrak{m}_{2}$. Thus we have

$$
\int_{\widetilde{\mathfrak{m}}}\left|S_{1}\left(\lambda_{1} \alpha\right) S_{2}\left(\lambda_{2} \alpha\right) S_{k}\left(\lambda_{3} \alpha\right)\right|^{2} K_{\tau}(\alpha) d \alpha \ll \tau X^{1+2 / k-2 /\left(7 m_{2}(k)\right)+\varepsilon} .
$$

Now the remaining work is to handle the range $\mathfrak{m}^{*}=\mathfrak{m} \backslash \widetilde{\mathfrak{m}}$. We have the following lemma.

Lemma 4.4. We have

$$
\int_{\mathfrak{m}^{*}}\left|S_{1}\left(\lambda_{1} \alpha\right) S_{2}\left(\lambda_{2} \alpha\right) S_{k}\left(\lambda_{3} \alpha\right)\right|^{2} K_{\tau}(\alpha) d \alpha \ll \tau X^{1+2 / k-1 / 7+\varepsilon} .
$$

Proof. Note that for any $\alpha \in \mathfrak{m}^{*}$, we have

$$
\left|S_{1}\left(\lambda_{1} \alpha\right)\right|>X^{3 / 7+\varepsilon} \text { and }\left|S_{2}\left(\lambda_{2} \alpha\right)\right|>X^{3 / 7+\varepsilon}
$$

We divide $\mathfrak{m}^{*}$ into disjoint sets $S\left(Z_{1}, Z_{2}, y\right)$, such that for $\alpha \in S\left(Z_{1}, Z_{2}, y\right)$, we have

$$
Z_{1}<\left|S_{1}\left(\lambda_{1} \alpha\right)\right| \leq 2 Z_{1}, \quad Z_{2}<\left|S_{2}\left(\lambda_{2} \alpha\right)\right| \leq 2 Z_{2}, \quad y<|\alpha| \leq 2 y
$$


where $Z_{1}=2^{k_{1}} X^{3 / 7+\varepsilon}, Z_{2}=2^{k_{2}} X^{3 / 7+\varepsilon}$ and $y=2^{k_{3}} X^{-3 / 4}$ for some non-negative integers $k_{1}, k_{2}, k_{3}$. Then from Lemma 4.1 , there exist two pairs of coprime integers $\left(a_{1}, q_{1}\right)$ and $\left(a_{2}, q_{2}\right)$ satisfying

$$
1 \leq q_{i} \ll\left(\frac{X^{1 / 2+\varepsilon / 2}}{Z_{i}}\right)^{4}, \quad\left|q_{i} \lambda_{i} \alpha-a_{i}\right| \ll X^{-1}\left(\frac{X^{1 / 2+\varepsilon / 2}}{Z_{i}}\right)^{4}, \quad i=1,2 .
$$

We remark that $a_{1} a_{2} \neq 0$, since otherwise we have $\alpha \in \mathfrak{M}$. Furthermore, we subdivide $S\left(Z_{1}, Z_{2}, y\right)$ into sets $S\left(Z_{1}, Z_{2}, y, Q_{1}, Q_{2}\right)$, where $Q_{j}<q_{j} \leq 2 Q_{j}$ on each set. Then we have

$$
\begin{aligned}
\left|a_{2} q_{1} \frac{\lambda_{1}}{\lambda_{2}}-a_{1} q_{2}\right| & =\left|\left(q_{1} \lambda_{1} \alpha-a_{1}\right) \frac{a_{2}}{\lambda_{2} \alpha}-\left(q_{2} \lambda_{2} \alpha-a_{2}\right) \frac{a_{1}}{\lambda_{2} \alpha}\right| \\
& \ll Q_{2} X^{-1}\left(\frac{X^{1 / 2+\varepsilon / 2}}{Z_{1}}\right)^{4}+Q_{1} X^{-1}\left(\frac{X^{1 / 2+\varepsilon / 2}}{Z_{2}}\right)^{4} \\
& \ll X^{3+4 \varepsilon} Z_{1}^{-4} Z_{2}^{-4} \\
& \ll X^{-3 / 7-4 \varepsilon} .
\end{aligned}
$$

Recall that $q=X^{3 / 7}$. Thus

$$
\left|a_{2} q_{1} \frac{\lambda_{1}}{\lambda_{2}}-a_{1} q_{2}\right|=o\left(q^{-1}\right)
$$

We also have $\left|a_{2} q_{1}\right| \ll y Q_{1} Q_{2}$. Thus, if $\left|a_{2} q_{1}\right|$ took $W$ distinct values, we could deduce the existence of $n$ satisfying

$$
\left\|n \frac{\lambda_{1}}{\lambda_{2}}\right\| \ll X^{-3 / 7-4 \varepsilon}, \quad n \ll \frac{y Q_{1} Q_{2}}{W} .
$$

This would contradict $a / q$ being a convergent to $\lambda_{1} / \lambda_{2}$ if $q$ is sufficiently large, unless

$$
W \ll \frac{y Q_{1} Q_{2}}{q} .
$$

From the upper bound for the divisor function, each value of $\left|a_{2} q_{1}\right|$ corresponds to $O\left(X^{\varepsilon}\right)$ values of $a_{2}, q_{1}$. Then we obtain that each set of $S\left(Z_{1}, Z_{2}, y, Q_{1}, Q_{2}\right)$ is made up of $O\left(W X^{\varepsilon}\right)$ intervals of length

$$
\begin{aligned}
& \ll \min \left(Q_{1}^{-1} X^{-1}\left(\frac{X^{1 / 2+\varepsilon / 2}}{Z_{1}}\right)^{4}, Q_{2}^{-1} X^{-1}\left(\frac{X^{1 / 2+\varepsilon / 2}}{Z_{2}}\right)^{4}\right) \\
& \ll \frac{X^{1+2 \varepsilon}}{Z_{1}^{2} Z_{2}^{2} Q_{1}^{1 / 2} Q_{2}^{1 / 2}} .
\end{aligned}
$$


Let $\mathcal{A}$ denote such a set $S\left(Z_{1}, Z_{2}, y, Q_{1}, Q_{2}\right)$. Then integrating over $\mathcal{A}$ gives

$$
\begin{aligned}
& \int_{\mathcal{A}}\left|S_{1}\left(\lambda_{1} \alpha\right) S_{2}\left(\lambda_{2} \alpha\right) S_{k}\left(\lambda_{3} \alpha\right)\right|^{2} K_{\tau}(\alpha) d \alpha \\
\ll & \min \left(\tau^{2}, y^{-2}\right) Z_{1}^{2} Z_{2}^{2} X^{2 / k} \frac{X^{1+2 \varepsilon}}{Z_{1}^{2} Z_{2}^{2} Q_{1}^{1 / 2} Q_{2}^{1 / 2}} \frac{X^{\varepsilon} y Q_{1} Q_{2}}{q} \\
\ll & \tau X^{1+2 / k-1 / 7+\varepsilon} .
\end{aligned}
$$

Then summing over all possible values of $Z_{1}, Z_{2}, y, Q_{1}, Q_{2}$, we conclude that

$$
\int_{\mathfrak{m}^{*}}\left|S_{1}\left(\lambda_{1} \alpha\right) S_{2}\left(\lambda_{2} \alpha\right) S_{k}\left(\lambda_{3} \alpha\right)\right|^{2} K_{\tau}(\alpha) d \alpha \ll \tau X^{1+2 / k-1 / 7+\varepsilon} .
$$

Recalling (4.1), from Lemmas 4.3 and 4.4 we can get the following lemma immediately.

Lemma 4.5. For $k \geq 3$, we have

$$
\int_{\mathfrak{m}}\left|H(\alpha) S_{3}\left(\lambda_{3} \alpha\right) S_{k}\left(\lambda_{4} \alpha\right)\right|^{2} K_{\tau}(\alpha) d \alpha \ll \tau X^{1+2 / k-2 /\left(7 m_{2}(k)\right)+\varepsilon},
$$

where $m_{2}(k)$ is defined by (1.1).

\section{The trivial arc}

In this section, we estimate the contribution of the right side of (2.6) from the trivial arc. Due to the similar reason as in Section 4, we also consider $S_{i}(\alpha)$ instead of $S_{i}\left(\alpha, \rho^{ \pm}\right)$, $i=1,2$ in the trivial arc. Applying the trivial bounds for $S_{k}\left(\lambda_{3} \alpha\right)$ and Cauchy's inequality, recalling $R=\tau^{-2} X^{1 / 2+2 \varepsilon}$ we have

$$
\begin{aligned}
& \int_{\mathfrak{t}}\left|S_{1}\left(\lambda_{1} \alpha\right) S_{2}\left(\lambda_{2} \alpha\right) S_{3}\left(\lambda_{3} \alpha\right) S_{k}\left(\lambda_{4} \alpha\right)\right| K_{\tau}(\alpha) d \alpha \\
\ll & X^{1 / k}\left(\int_{R}^{\infty}\left|S_{1}\left(\lambda_{1} \alpha\right)\right|^{2} K_{\tau}(\alpha) d \alpha\right)^{1 / 2}\left(\int_{R}^{\infty}\left|S_{2}\left(\lambda_{2} \alpha\right)\right|^{2} K_{\tau}(\alpha) d \alpha\right)^{1 / 2} \\
\ll & X^{1 / k}\left(\sum_{n=[R]}^{\infty} \int_{n}^{n+1}\left|S_{1}\left(\lambda_{1} \alpha\right)\right|^{2} \frac{1}{\alpha^{2}} d \alpha\right)^{1 / 2}\left(\sum_{n=[R]}^{\infty} \int_{n}^{n+1}\left|S_{2}\left(\lambda_{2} \alpha\right)\right|^{2} \frac{1}{\alpha^{2}} d \alpha\right)^{1 / 2} \\
\ll & X^{1 / k}\left(\sum_{n=[R]}^{\infty} \frac{1}{n^{2}}\right)\left(\int_{0}^{1}\left|S_{1}\left(\lambda_{1} \alpha\right)\right|^{2} d \alpha\right)^{1 / 2}\left(\int_{0}^{1}\left|S_{2}\left(\lambda_{2} \alpha\right)\right|^{2} d \alpha\right)^{1 / 2} \\
\ll & R^{-1} X^{1 / k+1 / 2+\varepsilon} \\
\ll & \tau^{2} X^{1 / k-\varepsilon} .
\end{aligned}
$$

Therefore we have

$$
\int_{\mathfrak{t}}\left|H(\alpha) S_{k}\left(\lambda_{3} \alpha\right)\right| K_{\tau}(\alpha) d \alpha \ll \tau^{2} X^{1 / k-\varepsilon}
$$




\section{Proof of Theorem 1.2}

In this section, we complete the proof of Theorem 1.2. Let $m_{2}(k)$ be defined as in (1.1). We take $\tau=X^{-\delta}$. Let $\mathcal{E}_{k}=\mathcal{E}_{k}(\mathcal{V}, X, \delta)$ denote the set of $v \in\left[\frac{1}{2} X, X\right] \cap \mathcal{V}$ such that the inequality (1.2) has no solution in primes $p_{1}, p_{2}, p_{3}$, and $E_{k}=E_{k}(\mathcal{V}, X, \delta)=\left|\mathcal{E}_{k}(\mathcal{V}, X, \delta)\right|$. Then from Lemma 3.1 and (5.1), we have

$$
\left|\sum_{v \in \mathcal{E}_{k}} \int_{\mathfrak{m}} H(\alpha) S_{k}\left(\lambda_{3} \alpha\right) e(-\alpha v) K_{\tau}(\alpha) d \alpha\right| \gg \tau^{2} X^{1 / k} L^{-2} E_{k} .
$$

Applying Cauchy's inequality, we have

$$
\begin{aligned}
& \left|\sum_{v \in \mathcal{E}_{k}} \int_{\mathfrak{m}} H(\alpha) S_{k}\left(\lambda_{3} \alpha\right) e(-\alpha v) K_{\tau}(\alpha) d \alpha\right| \\
& \ll\left(\int_{-\infty}^{+\infty}\left|\sum_{v \in \mathcal{E}_{k}} e(-\alpha v)\right|^{2} K_{\tau}(\alpha) d \alpha\right)^{1 / 2}\left(\int_{\mathfrak{m}}\left|H(\alpha) S_{k}\left(\lambda_{3} \alpha\right)\right|^{2} K_{\tau}(\alpha) d \alpha\right)^{1 / 2} \\
& \ll\left(\tau X^{1+2 / k-2 /\left(7 m_{2}(k)\right)+\varepsilon}\right)^{1 / 2}\left(\sum_{v_{1}, v_{2} \in \mathcal{E}_{k}} \max \left(0, \tau-\left|v_{1}-v_{2}\right|\right)\right)^{1 / 2} \\
& \ll \tau E_{k}^{1 / 2}\left(X^{1+2 / k-2 /\left(7 m_{2}(k)\right)+\varepsilon}\right)^{1 / 2} .
\end{aligned}
$$

Then combining 6.1) and 6.2), we have

$$
E_{k}\left(\mathcal{V}, X_{j}, \delta\right) \ll X_{j}^{1-2 /\left(7 m_{2}(k)\right)+2 \delta+\varepsilon} .
$$

Since $\lambda_{1} / \lambda_{2}$ is irrational, there are infinitely many $q$ we could have taken and this gives the sequence $X_{j} \rightarrow \infty$.

Now, if the convergent denominators for $\lambda_{1} / \lambda_{2}$ satisfy (1.5), then we can modify our works in Lemmas 4.3 and 4.4. We now assume that

$$
\min \left(Z_{1}, Z_{2}\right)>X^{\chi+\varepsilon}
$$

with $\chi$ given by (1.7). We then obtain

$$
\left|a_{2} q_{1} \frac{\lambda_{1}}{\lambda_{2}}-a_{1} q_{2}\right| \ll X^{3-8 \chi-4 \varepsilon} .
$$

However, we know from 1.5. that there is a convergent $a / q$ to $\lambda_{1} / \lambda_{2}$ with

$$
X^{(1-w)(8 \chi-3)} \ll q \ll X^{8 \chi-3} .
$$

The expression corresponding to 4.4 is now

$$
\begin{aligned}
& \int_{\mathcal{A}}\left|S_{1}\left(\lambda_{1} \alpha\right) S_{2}\left(\lambda_{2} \alpha\right) S_{k}\left(\lambda_{3} \alpha\right)\right|^{2} K_{\tau}(\alpha) d \alpha \\
\ll & \tau X^{3+2 / k-4 \chi+\varepsilon} q^{-1} \ll \tau X^{3+2 / k-4 \chi-(1-w)(8 \chi-3)+\varepsilon} \\
\ll & \tau X^{1+2 / k-(2-4 \chi) / m_{2}(k)+\varepsilon}
\end{aligned}
$$


by our choice of $\chi$. Thus

$$
\int_{\mathfrak{m}}\left|S_{1}\left(\lambda_{1} \alpha\right) S_{2}\left(\lambda_{2} \alpha\right) S_{k}\left(\lambda_{3} \alpha\right)\right|^{2} K_{\tau}(\alpha) d \alpha \ll \tau X^{1+2 / k-(2-4 \chi) / m_{2}(k)+\varepsilon} .
$$

Working as (6.1)-(6.3), we can complete the proof of Theorem 1.2 easily.

\section{Proof of Theorem 1.3}

In this section, we sketch the proof of Theorem 1.3 . We write

$$
\begin{array}{ll}
I_{1}^{\prime}=\left[\left(\frac{\eta X}{\lambda_{1}}\right)^{1 / 2},\left(\frac{2 \eta X}{\lambda_{1}}\right)^{1 / 2}\right], & I_{2}^{\prime}=\left[\left(\frac{\eta X}{\lambda_{2}}\right)^{1 / 2},\left(\frac{2 \eta X}{\lambda_{2}}\right)^{1 / 2}\right], \\
I_{3}^{\prime}=\left[\left(\frac{\eta X}{\lambda_{3}}\right)^{1 / 3},\left(\frac{2 \eta X}{\lambda_{3}}\right)^{1 / 3}\right], & I_{4}^{\prime}=\left[\left(\frac{\eta X}{\lambda_{4}}\right)^{1 / k},\left(\frac{2 \eta X}{\lambda_{4}}\right)^{1 / k}\right],
\end{array}
$$

and define

$$
\begin{aligned}
S_{1}^{\prime}(\alpha, \rho) & =\sum_{m \in I_{1}} \rho(m) e\left(m^{2} \alpha\right), & S_{2}^{\prime}(\alpha, \rho) & =\sum_{m \in I_{2}} \rho(m) e\left(m^{2} \alpha\right), \\
S_{3}^{\prime}(\alpha) & =\sum_{p \in I_{3}}(\log p) e\left(p^{3} \alpha\right), & S_{k}^{\prime}(\alpha) & =\sum_{p \in I_{4}}(\log p) e\left(p^{k} \alpha\right) .
\end{aligned}
$$

For any measurable subset $\mathfrak{X}$ of $\mathbb{R}$, write

$$
I^{\prime}\left(\tau, v, \mathfrak{X}, \rho_{1}, \rho_{2}\right)=\int_{\mathfrak{X}} S_{1}^{\prime}\left(\lambda_{1} \alpha, \rho_{1}\right) S_{2}^{\prime}\left(\lambda_{2} \alpha, \rho_{2}\right) S_{3}^{\prime}\left(\lambda_{3} \alpha\right) S_{k}^{\prime}\left(\lambda_{4} \alpha\right) e(-\alpha v) K_{\tau}(\alpha) d \alpha .
$$

Then from 2.2, we have

$$
\begin{aligned}
I^{\prime}\left(\tau, v, \mathbb{R}, \rho_{0}, \rho_{0}\right)= & \sum_{\substack{m_{i} \in I_{i}, i=1,2 \\
p_{j} \in I_{j}, j=3,4}} \rho_{0}\left(m_{1}\right) \rho_{0}\left(m_{2}\right)\left(\log p_{3}\right)\left(\log p_{4}\right) \\
& \quad \times \int_{-\infty}^{\infty} e\left(\left(\lambda_{1} m_{1}^{2}+\lambda_{2} m_{2}^{2}+\lambda_{3} p_{3}^{3}+\lambda_{4} p_{4}^{k}-v\right) \alpha\right) K_{\tau}(\alpha) d \alpha \\
= & \sum_{\substack{m_{i} \in I_{i}, i=1,2 \\
p_{j} \in I_{j}, j=3,4}} \rho_{0}\left(m_{1}\right) \rho_{0}\left(m_{2}\right) \log p_{3} \log p_{4} \\
& \quad \times \max \left(0, \tau-\left|\lambda_{1} m_{1}^{2}+\lambda_{2} m_{2}^{2}+\lambda_{3} p_{3}^{3}+\lambda_{4} p_{4}^{k}-v\right|\right) .
\end{aligned}
$$

Thus we have

$$
I^{\prime}\left(\tau, v, \mathbb{R}, \rho_{0}, \rho_{0}\right) \ll \tau(\log X)^{2} \mathfrak{N}^{\prime}(X),
$$

where $\mathfrak{N}^{\prime}(X)$ denotes the number of solutions of the inequality

$$
\left|\lambda_{1} p_{1}^{2}+\lambda_{2} p_{2}^{2}+\lambda_{3} p_{3}^{3}+\lambda_{4} p_{4}^{k}-v\right|<\tau
$$


with $p_{j} \in I_{j}$ for $1 \leq j \leq 4$. Recalling that 2.5 , we have

$$
I^{\prime}\left(\tau, v, \mathbb{R}, \rho_{0}, \rho_{0}\right) \geq I^{\prime}\left(\tau, v, \mathbb{R}, \rho^{-}, \rho^{+}\right)+I^{\prime}\left(\tau, v, \mathbb{R}, \rho^{+}, \rho^{-}\right)-I^{\prime}\left(\tau, v, \mathbb{R}, \rho^{+}, \rho^{+}\right) .
$$

Then it is sufficient to give a positive lower bound for the right side of (7.1). To do this, we divide the real line into the major arc $\mathfrak{M}^{\prime}$, the minor arc $\mathfrak{m}^{\prime}$ and the trivial arc $\mathfrak{t}^{\prime}$. We define

$$
\mathfrak{M}^{\prime}=\{\alpha:|\alpha| \leq P / X\}, \quad \mathfrak{m}^{\prime}=\{\alpha: P / X<|\alpha| \leq R\}, \quad \mathfrak{t}^{\prime}=\{\alpha:|\alpha|>R\}
$$

where $P=X^{8 /(11 k)-2 \varepsilon}$ and $R=\tau^{-2} X^{1 / 2+2 \varepsilon}$.

We write

$$
H^{\prime}(\alpha)=S_{1}^{\prime}\left(\lambda_{1} \alpha, \rho^{-}\right) S_{2}^{\prime}\left(\lambda_{2} \alpha, \rho^{+}\right)+S_{1}^{\prime}\left(\lambda_{1} \alpha, \rho^{+}\right) S_{2}^{\prime}\left(\lambda_{2} \alpha, \rho^{-}\right)-S_{1}^{\prime}\left(\lambda_{1} \alpha, \rho^{+}\right) S_{2}^{\prime}\left(\lambda_{2} \alpha, \rho^{+}\right) .
$$

As in Sections 3 and 5, we can estimate the integral on $\mathfrak{M}^{\prime}$ and $\mathfrak{t}^{\prime}$. Here we just state the following lemmas without proof.

Lemma 7.1. For $k \geq 3$, we have

$$
\int_{\mathfrak{M}^{\prime}} H^{\prime}(\alpha) S_{3}^{\prime}\left(\lambda_{3} \alpha\right) S_{k}^{\prime}\left(\lambda_{4} \alpha\right) e(-\alpha v) K_{\tau}(\alpha) d \alpha \gg c^{\prime} \tau^{2} X^{1 / 3+1 / k} L^{-2}
$$

where $c^{\prime}>0$ is an absolute constant.

Lemma 7.2. For $k \geq 3$, we have

$$
\int_{\mathfrak{t}^{\prime}}\left|H^{\prime}(\alpha) S_{3}^{\prime}\left(\lambda_{3} \alpha\right) S_{k}^{\prime}\left(\lambda_{4} \alpha\right)\right| K_{\tau}(\alpha) d \alpha \ll \tau^{2} X^{1 / 3+1 / k-\varepsilon} .
$$

For the minor arc, we follow the argument in Section 4. Also without loss of generality we only to consider the integral

$$
\int_{\mathfrak{m}}\left|S_{1}^{\prime}\left(\lambda_{1} \alpha\right) S_{2}^{\prime}\left(\lambda_{2} \alpha\right) S_{3}^{\prime}\left(\lambda_{3} \alpha\right) S_{k}^{\prime}\left(\lambda_{4} \alpha\right)\right|^{2} K_{\tau}(\alpha) d \alpha
$$

where $S_{i}^{\prime}\left(\lambda_{i} \alpha\right), i=1,2$ takes the form 4.3. Let $\widetilde{\mathfrak{m}}^{\prime}=\mathfrak{m}_{1}^{\prime} \cup \mathfrak{m}_{2}^{\prime}, \mathfrak{m}^{\prime *}=\mathfrak{m}^{\prime} \backslash \widetilde{\mathfrak{m}}^{\prime}$, where

$$
\mathfrak{m}_{j}^{\prime}=\left\{\alpha \in \mathfrak{m}^{\prime}:\left|S_{j}\left(\lambda_{j} \alpha\right)\right| \leq X^{3 / 7+\varepsilon}\right\} \quad \text { for } j=1,2
$$

Lemma 7.3. For $k \geq 3$, we have

$$
\int_{\widetilde{\mathfrak{m}^{\prime}}}\left|S_{1}^{\prime}\left(\lambda_{1} \alpha\right) S_{2}^{\prime}\left(\lambda_{2} \alpha\right) S_{3}^{\prime}\left(\lambda_{3} \alpha\right) S_{k}^{\prime}\left(\lambda_{4} \alpha\right)\right|^{2} K_{\tau}(\alpha) d \alpha \ll \tau X^{1+2 / 3+2 / k-\sigma^{*}(k)+\varepsilon},
$$

where $\sigma^{*}(k)$ is defined by 1.10 . 
Proof. We first consider the cases $k=3,4$. Using Hölder's inequality and Lemma 4.2, we can obtain

$$
\begin{aligned}
& \int_{\mathfrak{m}_{1}^{\prime}}\left|S_{1}^{\prime}\left(\lambda_{1} \alpha\right) S_{2}^{\prime}\left(\lambda_{2} \alpha\right) S_{3}^{\prime}\left(\lambda_{3} \alpha\right) S_{k}^{\prime}\left(\lambda_{4} \alpha\right)\right|^{2} K_{\tau}(\alpha) d \alpha \\
& \ll\left(\sup _{\alpha \in \mathfrak{m}_{1}^{\prime}}\left|S_{1}^{\prime}\left(\lambda_{1} \alpha\right)\right|\right)^{2}\left(\int_{\mathbb{R}}\left|S_{2}^{\prime}\left(\lambda_{2} \alpha\right)\right|^{4} K_{\tau}(\alpha) d \alpha\right)^{1 / 4}\left(\int_{\mathbb{R}}\left|S_{3}^{\prime}\left(\lambda_{3} \alpha\right)\right|^{8} K_{\tau}(\alpha) d \alpha\right)^{1 / 4} \\
& \quad \times\left(\int_{\mathbb{R}} \mid S_{2}^{\prime}\left(\left.\lambda_{2} \alpha\right|^{2}\left|S_{k}^{\prime}\left(\lambda_{4} \alpha\right)\right|^{4} K_{\tau}(\alpha) d \alpha\right)^{1 / 2}\right. \\
& \ll \\
& \ll X^{1+2 / 3+2 / k-1 / 7+\varepsilon} .
\end{aligned}
$$

Now we turn to handle the case $5 \leq k \leq 48$. Using Hölder's inequality and Lemma 4.2 again, we have

$$
\begin{aligned}
& \int_{\mathfrak{m}_{1}^{\prime}}\left|S_{1}^{\prime}\left(\lambda_{1} \alpha\right) S_{2}^{\prime}\left(\lambda_{2} \alpha\right) S_{3}^{\prime}\left(\lambda_{3} \alpha\right) S_{k}^{\prime}\left(\lambda_{4} \alpha\right)\right|^{2} K_{\tau}(\alpha) d \alpha \\
& \ll\left(\sup _{\alpha \in \mathfrak{m}_{1}{ }^{\prime}}\left|S_{1}^{\prime}\left(\lambda_{1} \alpha\right)\right|\right)^{1+2 / m_{3}(k)}\left(\int_{\mathbb{R}}\left|S_{1}^{\prime}\left(\lambda_{1} \alpha\right)\right|^{4} K_{\tau}(\alpha) d \alpha\right)^{1 / 4-1 /\left(2 m_{3}(k)\right)} \\
& \quad \times\left(\int_{\mathbb{R}}\left|S_{2}^{\prime}\left(\lambda_{2} \alpha\right)\right|^{4} K_{\tau}(\alpha) d \alpha\right)^{1 / 2-1 / m_{3}(k)}\left(\int_{\mathbb{R}}\left|S_{3}^{\prime}\left(\lambda_{3} \alpha\right)\right|^{8} K_{\tau}(\alpha) d \alpha\right)^{1 / 4-1 /\left(2 m_{3}(k)\right)} \\
& \quad \times\left(\int_{\mathbb{R}}\left|S_{2}^{\prime}\left(\lambda_{2} \alpha\right)\right|^{2}\left|S_{3}^{\prime}\left(\lambda_{3} \alpha\right)\right|^{2}\left|S_{k}^{\prime}\left(\lambda_{4} \alpha\right)\right|^{m_{3}(k)} K_{\tau}(\alpha) d \alpha\right)^{2 / m_{3}(k)} \\
& \ll \\
& \tau X^{1+2 / 3+2 / k-1 / 14-1 /\left(7 m_{3}(k)\right)+\varepsilon} .
\end{aligned}
$$

For $k \geq 49$, similarly we have

$$
\begin{aligned}
& \int_{\mathfrak{m}_{1}^{\prime}}\left|S_{1}^{\prime}\left(\lambda_{1} \alpha\right) S_{2}^{\prime}\left(\lambda_{2} \alpha\right) S_{3}^{\prime}\left(\lambda_{3} \alpha\right) S_{k}^{\prime}\left(\lambda_{4} \alpha\right)\right|^{2} K_{\tau}(\alpha) d \alpha \\
& \ll\left(\sup _{\alpha \in \mathfrak{m}_{1},}\left|S_{1}^{\prime}\left(\lambda_{1} \alpha\right)\right|\right)^{1+4 / m_{2}(k)}\left(\int_{\mathbb{R}}\left|S_{1}^{\prime}\left(\lambda_{1} \alpha\right)\right|^{4} K_{\tau}(\alpha) d \alpha\right)^{1 / 4-1 / m_{2}(k)} \\
& \quad \times\left(\int_{\mathbb{R}}\left|S_{2}^{\prime}\left(\lambda_{2} \alpha\right)\right|^{4} K_{\tau}(\alpha) d \alpha\right)^{1 / 2-1 / m_{2}(k)}\left(\int_{\mathbb{R}}\left|S_{3}^{\prime}\left(\lambda_{3} \alpha\right)\right|^{8} K_{\tau}(\alpha) d \alpha\right)^{1 / 4} \\
& \quad \times\left(\left.\int_{\mathbb{R}}\left|S_{2}^{\prime}\left(\lambda_{2} \alpha\right)\right|^{2} S_{k}^{\prime}\left(\lambda_{4} \alpha\right)\right|^{m_{2}(k)} K_{\tau}(\alpha) d \alpha\right)^{2 / m_{2}(k)} \\
& \ll \tau X^{1+2 / 3+2 / k-1 / 14-2 /\left(7 m_{2}(k)\right)+\varepsilon} .
\end{aligned}
$$

By symmetry we can get the same bound for the integral on $\mathfrak{m}_{2}^{\prime}$. Thus we have

$$
\int_{\widetilde{\mathfrak{m}}^{\prime}}\left|S_{1}^{\prime}\left(\lambda_{1} \alpha\right) S_{2}^{\prime}\left(\lambda_{2} \alpha\right) S_{3}^{\prime}\left(\lambda_{3} \alpha\right) S_{k}^{\prime}\left(\lambda_{4} \alpha\right)\right|^{2} K_{\tau}(\alpha) d \alpha \ll \tau X^{1+2 / 3+2 / k-\sigma *(k)+\varepsilon} .
$$

We handle $\mathfrak{m}^{\prime *}$ similarly to Lemma 4.4 and can get the following lemma. 
Lemma 7.4. We have

$$
\int_{\mathfrak{m}^{\prime *}}\left|S_{1}^{\prime}\left(\lambda_{1} \alpha\right) S_{2}^{\prime}\left(\lambda_{2} \alpha\right) S_{3}^{\prime}\left(\lambda_{3} \alpha\right) S_{k}^{\prime}\left(\lambda_{4} \alpha\right)\right|^{2} K_{\tau}(\alpha) d \alpha \ll \tau X^{1+2 / 3+2 / k-1 / 7+\varepsilon} .
$$

Due to a similar reason to 4.1), from Lemmas 7.3 and 7.4 we can deduce that

Lemma 7.5. For $k \geq 3$, we have

$$
\int_{\mathfrak{m}^{\prime}}\left|H^{\prime}(\alpha) S_{3}^{\prime}\left(\lambda_{3} \alpha\right) S_{k}^{\prime}\left(\lambda_{4} \alpha\right)\right|^{2} K_{\tau}(\alpha) d \alpha \ll \tau X^{1+2 / 3+2 / k-\sigma *(k)+\varepsilon},
$$

where $\sigma^{*}(k)$ is defined by 1.10 .

Then from Lemmas 7.1, 7.2 and 7.5, arguing similarly to (6.1)-(6.3), we can complete the proof of Theorem 1.3 easily.

\section{Acknowledgments}

The authors would like to thank the referee for useful comments and corrections on the manuscript. The authors appreciate Professor Angel Kumchev for valuable suggestions on the original manuscript. This work is supported by the Natural Science Foundation of Shandong Province (Grant No. ZR2018BA006) and the National Natural Science Foundation of China (Grant No. 11801328).

\section{References}

[1] J. Bourgain, C. Demeter and L. Guth, Proof of the main conjecture in Vinogradov's mean value theorem for degrees higher than three, Ann. of Math. (2) 184 (2016), no. 2, 633-682.

[2] J. Brüdern and É. Fouvry, Lagrange's four squares theorem with almost prime variables, J. Reine Angew. Math. 454 (1994), 59-96.

[3] H. Davenport, Analytic Methods for Diophantine Equations and Diophantine Inequalities, Second edition, Cambridge Mathematical Library, Cambridge University Press, Cambridge, 2005.

[4] G. Gao and Z. Liu, Results of Diophantine approximation by unlike powers of primes, Front. Math. China 13 (2018), no. 4, 797-808.

[5] W. Ge and T. Wang, On Diophantine problems with mixed powers of primes, Acta Arith. 182 (2018), no. 2, 183-199. 
[6] G. Harman, The values of ternary quadratic forms at prime arguments, Mathematicka 51 (2004), no. 1-2, 83-96.

[7] G. Harman and A. V. Kumchev, On sums of squares of primes, Math. Proc. Cambridge Philos. Soc. 140 (2006), no. 1, 1-13.

[8] W. P. Li and T. Z. Wang, Diophantine approximation with prime variables and mixed powers, Chinese Ann. Math. Ser. A 31 (2010), no. 2, 247-256.

[9] Q. W. Mu and X. D. Lü, Diophantine approximation with prime variables and mixed powers, Chinese Ann. Math. Ser. A 36 (2015), no. 3, 303-312.

[10] Diophantine approximation with prime variables and mixed powers (II), Adv. Math. (China) 46 (2017), no. 2, 190-202.

[11] E. C. Titchmarsh, The Theory of the Riemann Zeta-function, Second edition, Oxford University Press, Oxford, 1986.

[12] R. C. Vaughan, Diophantine approximation by prime numbers II, Proc. London Math. Soc. (3) 28 (1974), 385-401.

[13] Y. Wang and W. Yao, Diophantine approximation with one prime and three squares of primes, J. Number Theory 180 (2017), 234-250.

Huafeng Liu and Jing Huang

School of Mathematics and Statistics, Shandong Normal University, Jinan, Shandong 250014, China

E-mail address: hfliu_sdu@hotmail.com, huangjingsdnu@163.com 